\title{
Uso de marcapasos con sensor de asa cerrada en pacientes con síncope vasovagal refractario al tratamiento médico
}

\author{
Mauricio Duque ${ }^{\mathrm{a}, *}$, Martín R. de la Ossa ${ }^{\mathrm{a}}$, Juan C. Díaz ${ }^{\mathrm{a}}$, Jorge E. Marín ${ }^{\mathrm{b}}$, \\ Laura Duque $^{\mathrm{a}}$, Jorge E. Velásquez ${ }^{\mathrm{a}}$, Julián M. Aristizábal ${ }^{\mathrm{a}}$, \\ Luiz F. Vassão Jr. ${ }^{\mathrm{c}}$ y William Uribe ${ }^{\mathrm{a}}$
}

a Cardiología y Electrofisiología, CES Cardiología, Medellín, Colombia

b Universidad CES, Medellín, Colombia

c BIOTRONIK Latin America

Recibido el 14 de enero de 2015; aceptado el 16 de julio de 2015

Disponible en Internet el 31 de octubre de 2015

\section{PALABRAS CLAVE \\ Marcapasos; \\ Síncope vasovagal; \\ Estimulación \\ ventricular}

\begin{abstract}
Resumen
Introducción: Estudios previos no han encontrado un beneficio claro relacionado con el uso de marcapasos en síncope cardioinhibitorio; sin embargo, la mayoría de estos fueron realizados con marcapasos con algoritmos de estimulación basados en la caída abrupta de la frecuencia cardíaca.

Objetivo: Evaluar el impacto de la estimulación cardiaca utilizando marcapasos con sensor de asa cerrada en el número de síncopes e impacto en la calidad de vida de los pacientes con síncope vasovagal refractario al manejo médico.

Métodos: Se registraron de manera prospectiva todos los pacientes con diagnóstico de síncope vasovagal refractario a manejo médico a quienes se les implantó un marcapasos bicameral con sensor de asa cerrada durante el periodo comprendido entre agosto de 2008 y agosto de 2013. Se evaluó la frecuencia de eventos sincopales y la calidad de vida (por medio del cuestionario SFSQ) antes y después del implante del dispositivo.

Resultados: Se incluyeron 51 pacientes (27 mujeres, 53\%), con promedio de edad de $52,98 \pm 16,88$ años y un seguimiento de $20,19 \pm 14,64$ meses. Se registraron 30 casos $(58,8 \%)$ de síncope cardioinhibitorio, $20(39,21 \%)$ de mixto y $1(1,9 \%)$ de vasodepresor. El número de síncopes posterior al implante del dispositivo con sensor de asa cerrada, disminuyó en forma estadísticamente significativa. Se registraron un total de 198 síncopes antes del implante del marcapasos, valor que descendió a 10 luego del implante (reducción del 94,9\%). Los pacientes experimentaron $3,88 \pm 3,52$ síncopes previo al implante, los cuales disminuyeron hasta $0,19 \pm 0,63$ episodios postimplante $(p<0,001)$. La calidad de vida mejoró después del implante:
\end{abstract}

\footnotetext{
* Autor para correspondencia.

Correo electrónico: mauricioduque@une.net.co (M. Duque).
} 
el puntaje promedio SFSQ por paciente preimplante, fue de $70,19 \pm 24,5$ vs. $15,88 \pm 13,73$ postimplante $(p=0,000)$.

Conclusión: El implante de un marcapasos con sensor de asa cerrada en pacientes con síncope vasovagal refractario a manejo médico, mostró una disminución significativa en el número de síncopes y mejoría en la calidad de vida.

(c) 2015 Sociedad Colombiana de Cardiología y Cirugía Cardiovascular. Publicado por Elsevier España, S.L.U. Este es un artículo Open Access bajo la licencia CC BY-NC-ND (http://creativecommons.org/licenses/by-nc-nd/4.0/).

\section{KEYWORDS}

Pacemaker; Vasovagal syncope; Ventricular stimulation
Use of closed loop sensor pacing system in patients with vasovagal syncope refractory to medical treatment

\begin{abstract}
Introduction: Previous studies have not found a clear benefit related to the use of pacemakers in cardioinhibitory syncopes; however, most of them were conducted with pacing systems using stimulation algorithms based in the abrupt drop of the heart rate.

Objetive: To assess the impact of cardiac stimulation using a closed loop sensor pacemaker in the number of syncopes and its impact in the quality of life of patients with a vasovagal syncope refractory to medical treatment.

Methods: Prospective registration of patients diagnosed with vasovagal syncope refractory to medical treatment, who were implanted a bicameral closed loop sensor pacemaker between August 2008 and August 2013. Frequency of syncopal events and quality of life (using the SFSQ) were assessed before and after implanting the device.

Results: 51 patients were included ( 27 women, $53 \%$ ), with an average age of $52.98 \pm 16.88$ and a follow-up of $20.19 \pm 14.64$ months. 30 cases $(58.8 \%)$ of cardioinhibitory syncope were registered, $20(39.21 \%)$ were mixed and $1(1.9 \%)$ was vasodepressor. The number of syncopes after the implantation of the closed loop sensor device was significantly reduced. Before the pacemaker was implanted, the total of registered syncopes was 198, a value that was reduced to 10 after the implantation (a $94.9 \%$ drop). Patients experienced $3.88 \pm 3.52$ before the implantation, and they decreased to $0.19 \pm 0.63$ episodes postimplant $(p<0.001)$. Quality of life improved after the implantation: patient average SFSQ score before the implantation was of $70.19 \pm 24.5$ vs. $15.88 \pm 13.73$ postimplant $(p=0.000)$.

Conclusion: Implanting a closed loop sensor pacemaker in patients with vasovagal syncope refractory to medical treatment revealed a significant decrease in the number of syncopes and an improvement in the quality of life.

(c) 2015 Sociedad Colombiana de Cardiología y Cirugía Cardiovascular. Published by Elsevier España, S.L.U. This is an open access article under the CC BY-NC-ND license (http://creativecommons.org/licenses/by-nc-nd/4.0/).
\end{abstract}

\section{Introducción}

El síncope es una pérdida súbita de conciencia secundaria a hipoperfusión cerebral transitoria, caracterizado por inicio rápido, corta duración y recuperación completa y espontánea ${ }^{1}$. Esta patología implica una carga considerable para los sistemas de salud del mundo, con una prevalencia que aumenta con la edad y va desde 6,2 por 1.000 personas/año en adultos jóvenes, hasta 19 por 1.000 personas/año en mayores de 80 años $^{2,3}$.

El síncope vasovagal es causado por un deterioro agudo de las interacciones entre el sistema nervioso autónomo y el cardiovascular, encargados de mantener la perfusión cerebral, lo cual lleva a una activación inapropiada del reflejo de Bezold-Jarisch por la estimulación de los mecanorreceptores. Este hecho representa un mecanismo combinado de vasodepresión y cardioinhibición, en el que, en la respuesta cardioinhibitoria previa al síncope, hay un incremento del estímulo simpático, y luego un retiro abrupto del parasimpático, que produce bradicardia o asistolia y posteriormente aparece el mecanismo compensatorio del simpático con el cual se incrementan la tensión arterial y la frecuencia cardíaca $^{4,5}$.

La estrategia inicial de manejo es conservadora, con énfasis en los cambios en el estilo de vida, en la minimización de las situaciones desencadenantes, así como en el reconocimiento temprano de los síntomas y el uso de maniobras para abortarlos ${ }^{6,7}$. En pacientes con síncope vasovagal donde la respuesta es vasodilatadora, la estimulación cardíaca no sería efectiva para prevenir la recurrencia del síncope; sin embargo, en los casos de respuesta mixta, con asistolia prolongada, la estimulación cardíaca pudiera ofrecer algunas ventajas ${ }^{4}$. Sobre este punto en particular, existe controversia en la literatura médica ${ }^{8}$, pues los resultados de los primeros estudios observacionales mostraron beneficio en la reducción del número de eventos 
sincopales mas no en los síntomas vasovagales ${ }^{9-12}$. Estos beneficios no lograron reproducirse en estudios controlados con placebo e incluso uno de estos debió ser suspendido por los pobres resultados ${ }^{13-15}$. Estos estudios emplearon sensores convencionales con detección de caída de la frecuencia cardíaca. Sin embargo al revisar la fisiopatología del síncope vasovagal, el paso inicial es un estímulo transitorio del simpático y posteriormente se desencadena la caída de la presión arterial y de la frecuencia cardíaca; por tanto, los marcapasos con sensores convencionales actúan al final del reflejo, mientras que el sensor de asa cerrada lo hace desde el comienzo ${ }^{4}$. Los marcapasos con asa cerrada (CLS, su siglas en inglés), miden la impedancia ventricular latido a latido, cualidad que les permite detectar cambios tempranos en la contractilidad (estímulo simpático). La propiedad de revelar variaciones precoces de la contractilidad cardíaca que suceden antes de la caída de la presión y del reflejo cardioinhibitorio, les permite a los marcapasos con CLS intervenir en forma más oportuna en comparación con otro tipo de sensores, manteniéndose así el gasto cardíaco y evitando el síncope ${ }^{16}$.

Estudios como el INVASY (INotropy controlled pacing in VAsovagal SYncope) y registros como los de Palmisano y Bortnik $^{17-19}$, han arrojado resultados alentadores con este tipo de dispositivos.

El objetivo del estudio que aquí se publica, fue determinar la utilidad del marcapasos con CLS en pacientes con síncope vasovagal, refractarios al manejo convencional.

\section{Métodos}

Se incluyeron pacientes con síncope vasovagal refractario (definido como la persistencia de eventos sincopales con limitación en la calidad de vida a pesar de manejo no farmacológico) mayores de 40 años, con más de dos eventos sincopales en el último año y una prueba de mesa basculante positiva para síncope vasovagal. Se excluyeron pacientes con bloqueo auriculoventricular de segundo o tercer grado, disfunción sinusal, taquiarritmias documentadas y falla cardiaca. Todos firmaron el consentimiento informado por escrito y a todos se les implantó un marcapasos bicameral con CLS (referencia: CYLOS DR o EVIA DR; Biotronik SE \& Co. KG, Berlín, Alemania), los cuales fueron programados en modo DDD-CLS.

Se obtuvieron datos basales antes del implante del marcapasos (presencia de comorbilidades, tratamiento recibido para el manejo de su síncope, número de síncopes durante el año anterior y medición de calidad de vida con el cuestionario SFSQ); posteriormente, se hicieron consultas de reprogramación, la primera a las 6 semanas del implante y posteriormente cada 6 meses; se realizaron además llamadas telefónicas de seguimiento cada 3 meses. No se les informó a los pacientes el objetivo del cuestionario SFSQ. Para el análisis estadístico, las variables cuantitativas se resumieron mediante el cálculo de sus medias, desviaciones estándar, medianas y rangos intercuartiles. Se usó la prueba de Kolmogorov-Smirnov para verificar normalidad; las diferencias antes y después del implante del marcapasos con algoritmo CLS, se establecieron con la prueba de Wilcoxon. Para las variables cualitativas se crearon proporciones. Los datos fueron procesados con el programa Stata versión

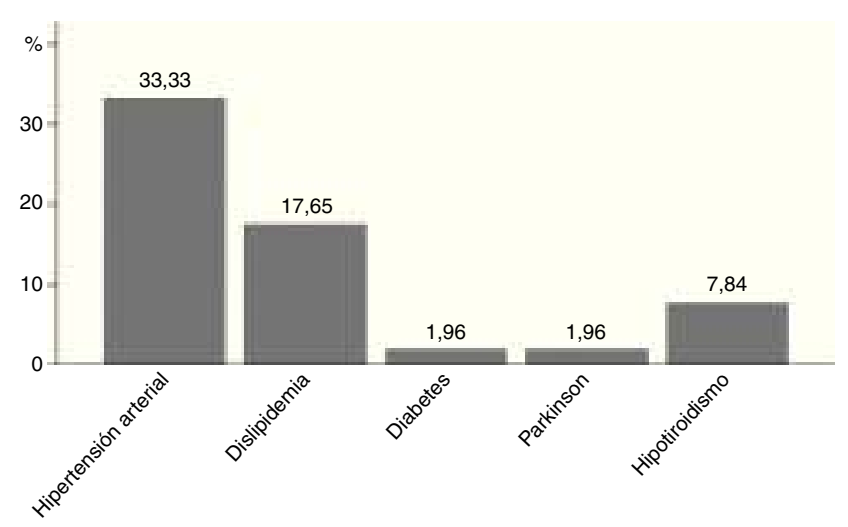

Figura 1 Comorbilidades.

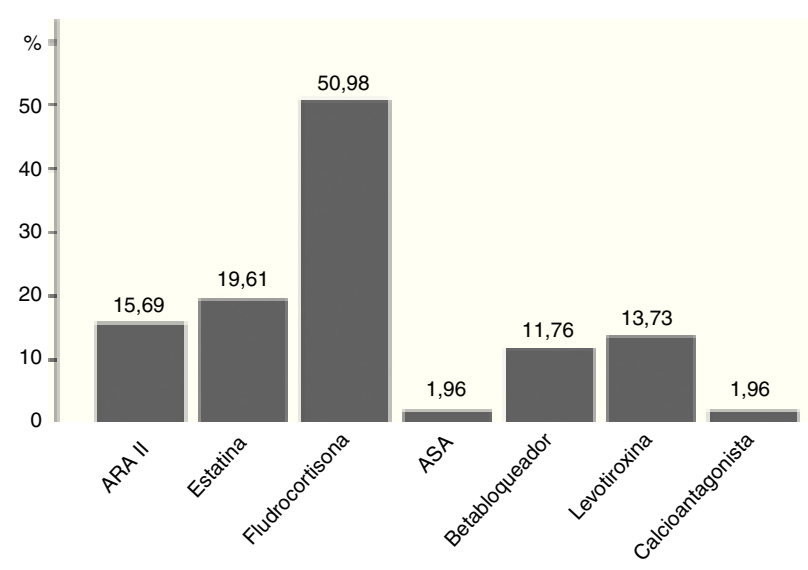

Figura 2 Medicamentos usados.

11 (StataCorp). Se trabajó con una significancia menor de 0,05 .

\section{Resultados}

Entre agosto de 2008 y agosto de 2013, se implantaron marcapasos Biotronik con sensor CLS a 51 pacientes ( 24 hombres, $47 \%$ ), con una edad promedio de $52,98 \pm 16,88$ años y un tiempo de seguimiento de 20,19 $\pm 14,64$ meses (rango: 1-48 meses). La hipertensión arterial fue la comorbilidad predominante y el $50,98 \%$ recibía tratamiento con fludrocortisona. Los tipos de síncope fueron: cardioinhibitorio en 30 casos $(58,8 \%)$, mixto en $20(39,21 \%)$ y vasodepresor en uno $(1,9 \%)$. En las figuras 1 y 2 se muestra la frecuencia de otras condiciones y medicamentos.

Se registraron en total 198 síncopes antes del implante del marcapasos, valor que descendió a 10 (reducción absoluta del 94,9\%) posterior al uso del dispositivo CLS. Esta reducción fue evidente en todos los participantes (figs. 3 y 4 ) En la fig. 7 se documenta el estimador de Kaplan-Meier de recurrencia del primer síncope después del implante del marcapasos con CLS. Se observó adicionalmente un incremento significativo en la calidad de vida posterior al implante del marcapasos para el total de la muestra y para ambos géneros (figs. 4 y 5). Estas diferencias se mantuvieron en el grupo de pacientes con síncopes cardioinhibitorio y mixto (tabla 1). 


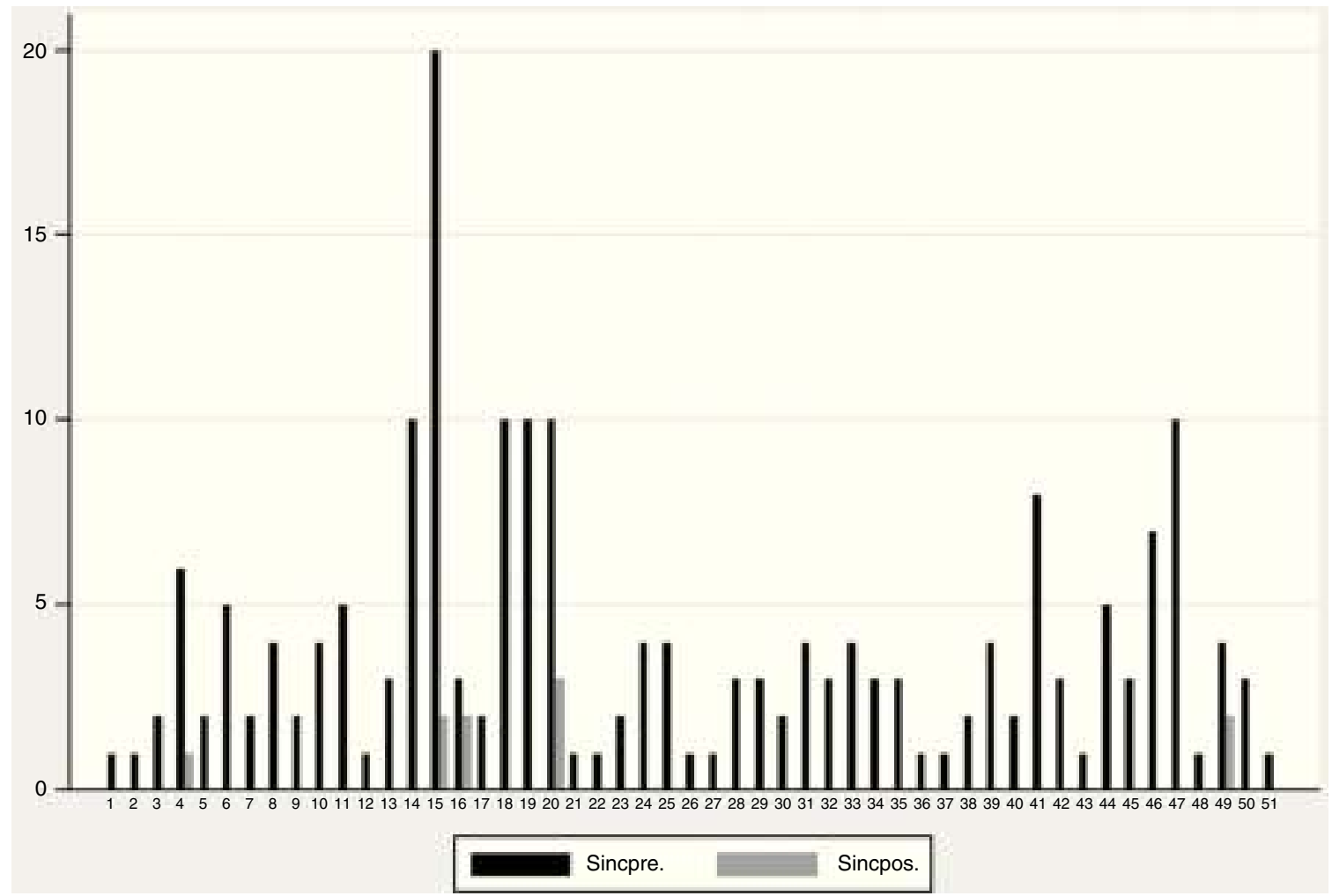

Figura 3 Comparación en frecuencia de síncopes por paciente.
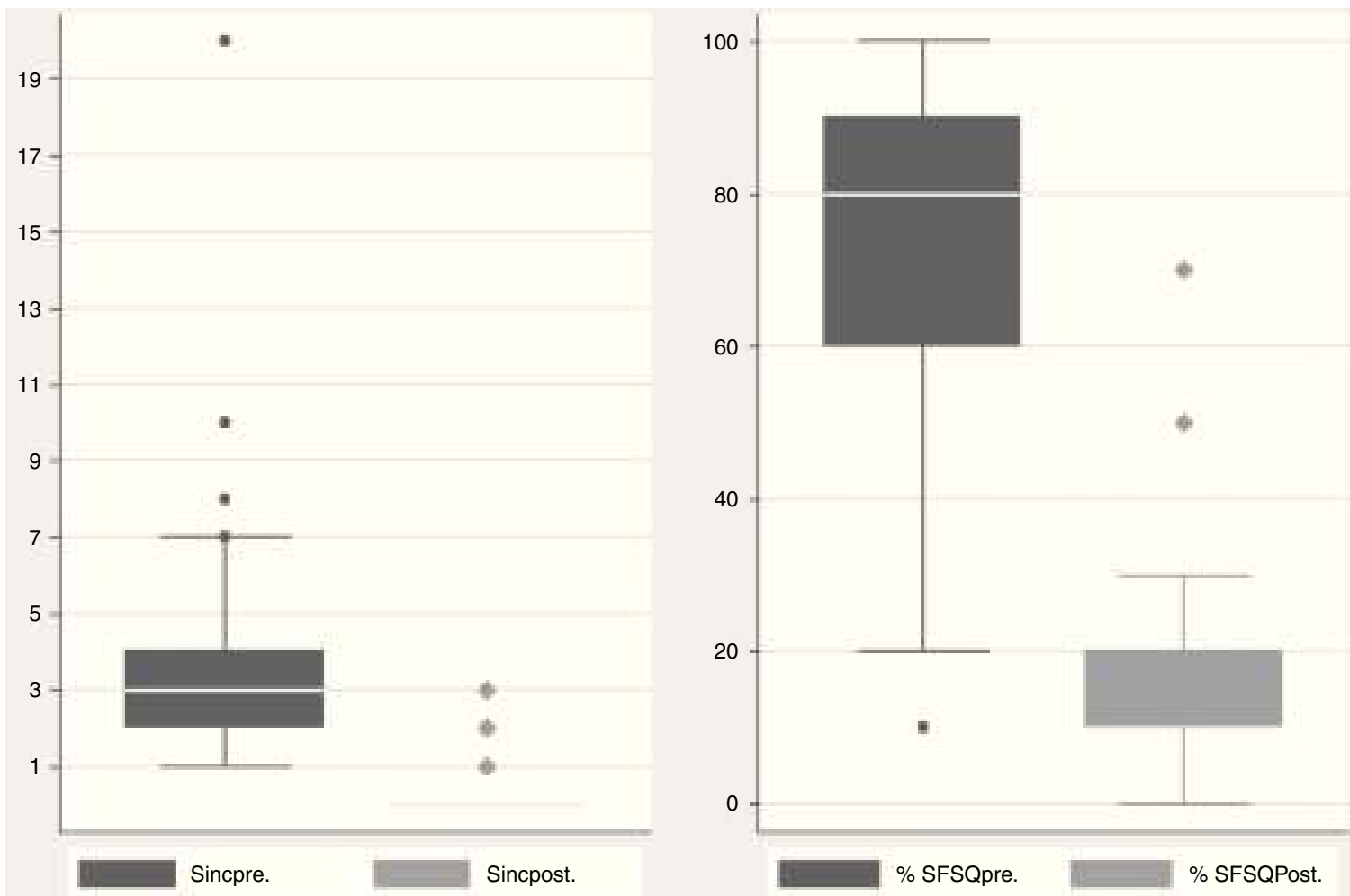

Figura 4 Comparación en frecuencia de síncopes y calidad de vida. 
Tabla 1 Frecuencia de síncopes

\begin{tabular}{|c|c|c|c|c|c|c|}
\hline Tipo de síncope & $\begin{array}{l}\text { Síncopes Pre } \\
\text { CLS }^{*}\end{array}$ & $\begin{array}{l}\text { Síncopes } \\
\text { post CLS }\end{array}$ & $p$ & $\begin{array}{l}\text { Calidad de } \\
\text { vida pre CLS }\end{array}$ & $\begin{array}{l}\text { Calidad de } \\
\text { vida post } \\
\text { CLS }\end{array}$ & $p$ \\
\hline Cardioinhibitorio (30) & $3,81 \pm 0,75(4)$ & $0(0)$ & 0,00 & $68,18 \pm 26(80)$ & $10,90 \pm 8,31(10)$ & 0,00 \\
\hline Mixto $(20)$ & $6 \pm 3,20\left(5^{* *}\right)$ & $0,33 \pm 0,70(0)$ & 0,007 & $84,44 \pm 16,66(90)$ & $17,77 \pm 22,79(20)$ & 0,007 \\
\hline Vasodepresor (1) & 2 & 0 & & 70 & 10 & \\
\hline
\end{tabular}

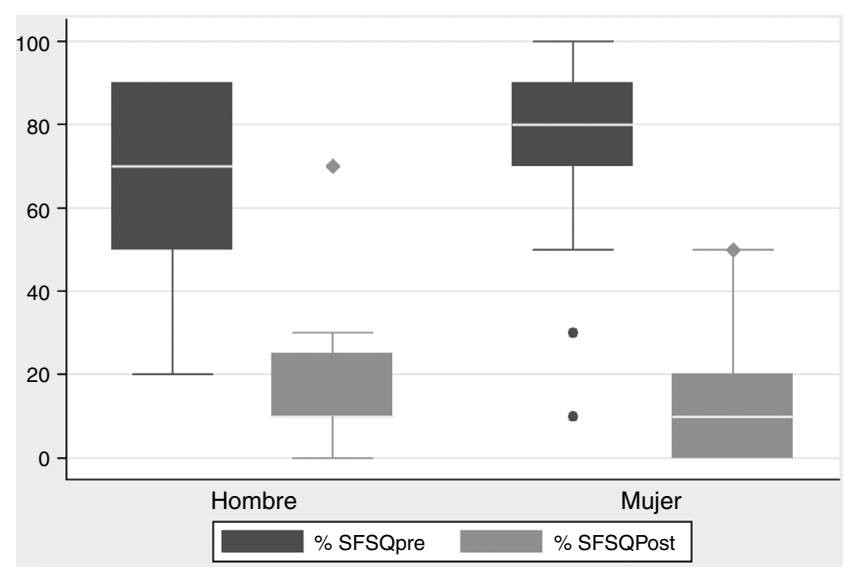

Figura 5 Comparación calidad de vida por género.

\section{Discusión}

La estimulación cardiaca permanente es una forma aun controversial de tratamiento en pacientes con síncope vasovagal, encaminada al manejo de la bradicardia transitoria que se produce durante el episodio sincopal, de modo que se provee una frecuencia cardíaca para soportar la caída de la presión arterial durante el evento ${ }^{5}$. A principios de los noventa se realizaron los primeros intentos de tratamiento con estimulación cardiaca en pacientes con síntomas refractarios al manejo médico bajo la premisa de la existencia de un componente cardioinhibitorio ${ }^{10}$.

Estos primeros estudios fueron sin distribución aleatoria, observacionales y en su mayoría terminaron de manera precoz debido a que los análisis preliminares mostraban excelentes resultados ${ }^{10-12}$. Estudios posteriores en los que se usó sensor convencional con detección de caída de frecuencia, no mostraron resultados similares.

El papel del CLS en la prevención del síncope vasovagal, fue sugerido en el estudio INVASY, que reunió 50 pacientes, con al menos cinco episodios sincopales en los últimos dos años y una prueba de mesa basculante positiva para síncope cardioinhibitorio, quienes fueron distribuidos de manera aleatoria a estimulación DDD-CLS vs. DDI. Se encontró una recurrencia de síncope al año en el grupo de CLS de $0 \%$, frente a $78 \%$ en el grupo de $\mathrm{DDI}^{17}$. El estudio fue suspendido luego de doce meses, a la luz de estos resultados. La principal limitante del estudio INVASY fue el seguimiento relativamente corto, ya que los posibles periodos asintomáticos largos de los pacientes con síncope, son un factor que puede modificar la magnitud de los hallazgos ${ }^{17}$. Entre tanto, el registro de Palmisano con 41 pacientes con síncope vasovagal con respuesta cardioinhibitoria en la mesa basculante tratados con marcapasos con CLS en un seguimiento de ocho años, mostró recurrencia de síncope del $4 \%$ en el grupo de intervención contra el $40 \%$ en el grupo de estimulación convencional, sin evaluación del impacto sobre la calidad de vida de los pacientes ${ }^{18}$. Por su parte, Kanjwal et al. en una muestra de 35 pacientes con síncope vasovagal recurrente y con prueba de mesa basculante positiva para respuesta cardioinhibitoria, compararon la efectividad del CLS contra un sensor convencional y hallaron una recurrencia de síncopes del $59 \%$ vs. $83 \%$ a favor del sistema $\mathrm{CLS}^{20}$. La eficacia del CLS en el manejo del síncope vasovagal también fue documentada por Bortnik et al. en un registro en el que se incluyeron 35 pacientes con síncope y presíncope, con un promedio de seis episodios antes del implante del dispositivo. En el seguimiento, 29 de 35 pacientes (83\%) permancecieron asintomáticos y uno reportó persistencia del síncope pero mejoría en la calidad de vida ${ }^{19}$. En todos los pacientes el número de síncopes posterior al implante del CLS, fue significativamente menor.

El estudio más reciente con empleo de marcapasos con CLS para el tratamiento del síncope vasovagal con respuesta cardioinhibitoria en la mesa basculante, fue realizado en una población de pacientes sin otras comorbilidades, con un diseño crossover, en el que involucraron 50 pacientes con un seguimiento de 36 meses, y encontraron no solo una reducción significativa en el número de síncopes sino en el de presíncopes ${ }^{21}$.

El estudio que aquí se reporta constituye uno de los más grandes publicados a la fecha con este tipo de sensores. Es de diseño observacional prospectivo, compuesto por una muestra de 51 pacientes con síncope vasovagal, sin respuesta al manejo convencional, quienes fueron tratados con marcapasos con CLS. Los resultados obtenidos en cuanto a la disminución en el número de síncopes, fueron similares a los descritos en el INVASY y en los registros de Palmisano y de Bortnik. Un aporte adicional del estudio es la medición en la mejoría de la calidad de vida de los pacientes, realizada por medio de la escala SFSQ (Syncope Functional Status Questionnaire), la cual ha sido validada en un estudio de más de 500 pacientes $^{22}$. Lo anterior demuestra no solo el impacto que tiene el dispositivo en el número de síncopes sino también en la calidad de vida de los pacientes. En este sentido, es de resaltar que se incluyeron no solo pacientes con respuesta cardioinhibitoria, sino con respuesta mixta, en quienes se encontraron resultados similares. Así mismo, 


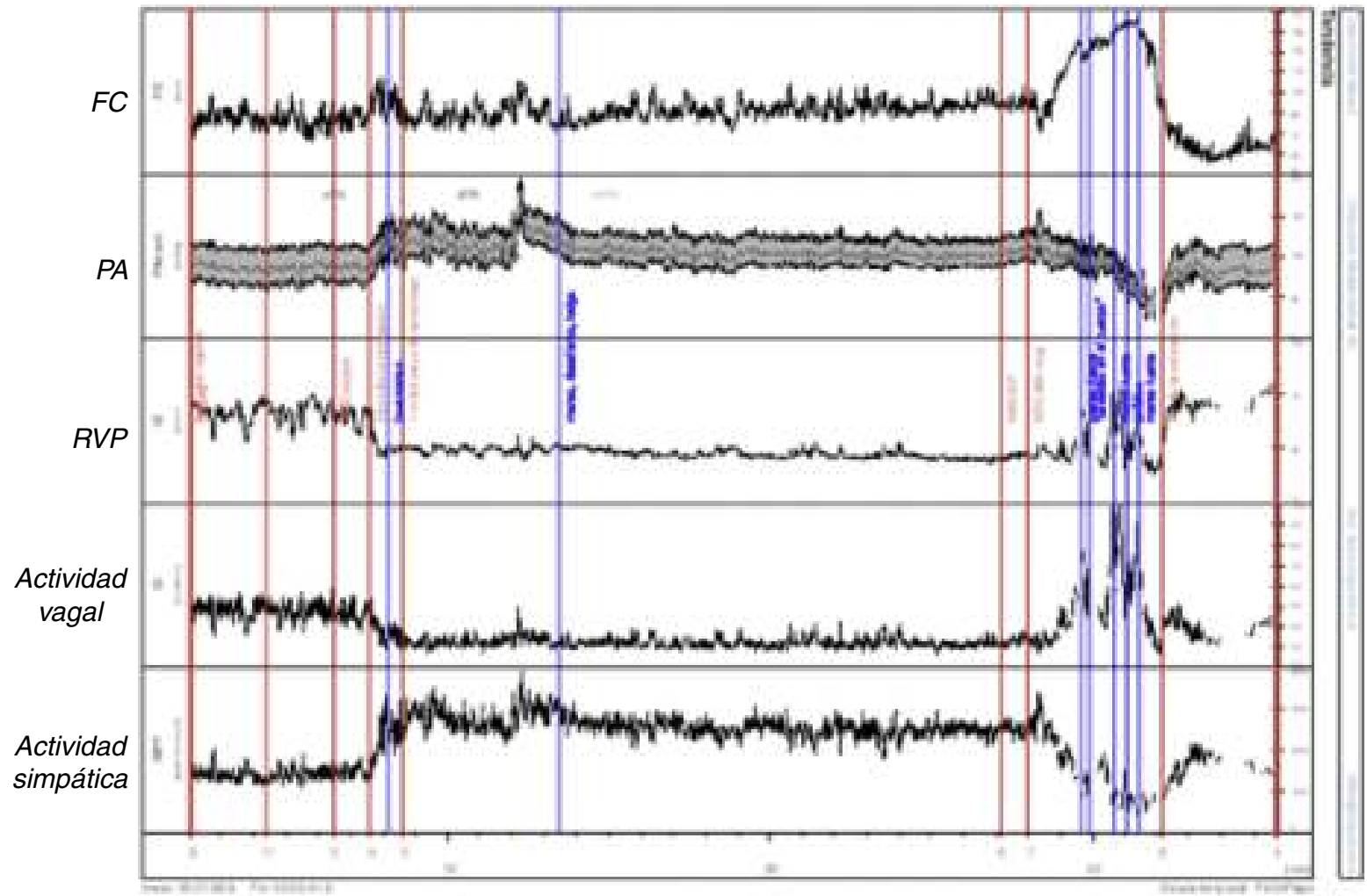

Figura 6 Explicación del fenómeno de estímulo del sensor CLS con el incremento del simpático.

se incluyó un paciente con respuesta vasodepresora, por marcado deterioro de la calidad de vida y ausencia de respuesta a la medicación y a las medidas no farmacológicas, quien tuvo mejoría de los síntomas.

En lo concerniente a las limitaciones del estudio, se trata de una muestra pequeña de pacientes lo cual puede sobreestimar el efecto de la intervención; sin embargo es comparable a los estudios de mayor trascendencia sobre el tema ${ }^{17-19}$. Es, igualmente, un estudio en el cual no se cotejó la intervención contra placebo. No obstante, los mismos pacientes constituyen el grupo control lo cual permite establecer diferencias significativas en el número de síncopes y en la calidad de vida.

Esta explicación está documentada en un test de mesa basculante, en el que se observa que antes de que se retire el simpático de forma abrupta y se estimule el parasimpático para que se produzca bradicardia y vasodilatación, es, en última instancia, lo que disminuye la perfusión cerebral y produce el síncope. En la figura 6 se observa con claridad que se produce un importante estímulo previo del simpático, en el que hay taquicardia y aumento de las resistencias periféricas (se activa el CLS) lo que evitaría la retirada abrupta del parasimpático, al menos de su componente de disminución de la frecuencia cardíaca. Esto fenómeno podría explicar por qué este sensor es mejor que otros que usan otros marcapasos para este tipo de patología.

Dada la naturaleza evidente del síncope, la magnitud en la diferencia hallada y la concordancia con los resultados de otros estudios, los autores creen que las diferencias en el número de síncopes antes y después del implante del marcapasos con CLS, se mantienen y sigue siendo significativas,

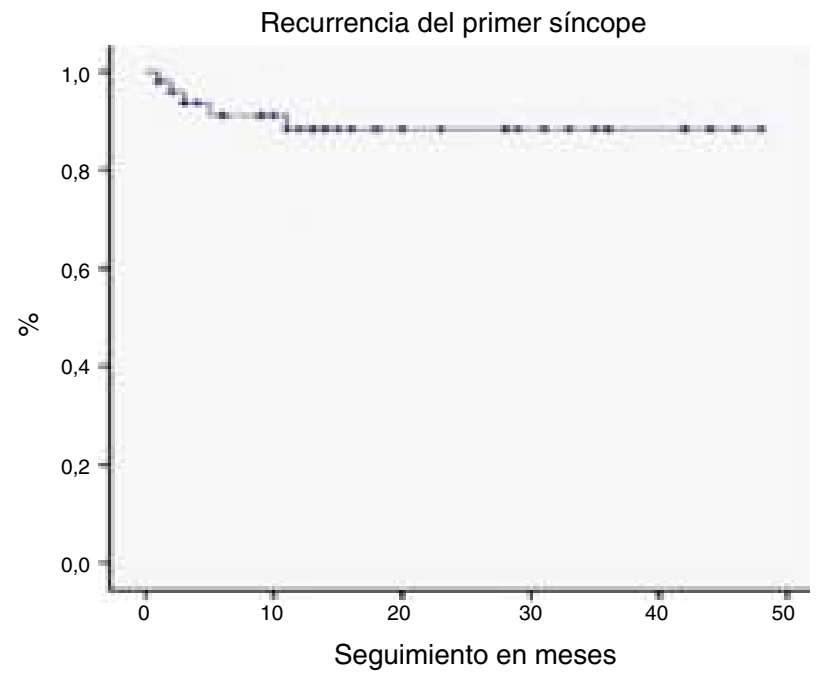

Figura 7 Curva de Kaplan-Meier de recurrencia de primer síncope.

lo cual les confiere a estos dispositivos la alternativa de considerarlos como una estrategia adicional en el manejo del paciente con síncope refractario al tratamiento.

\section{Conclusión}

El implante de un marcapasos con CLS en pacientes con síncope vasovagal ofrece una opción de manejo no solo para 
el control del número de síncopes sino para la mejoría de la calidad de vida de la población en mención. Se requieren estudios controlados con distribución aleatoria con enmascaramiento con mayor número de pacientes para corroborar el verdadero impacto de esta intervención.

\section{Responsabilidades éticas}

Protección de personas y animales. Los autores declaran que para esta investigación no se han realizado experimentos en seres humanos ni en animales.

Confidencialidad de los datos. Los autores declaran que en este artículo no aparecen datos de pacientes.

Derecho a la privacidad y consentimiento informado. Los autores declaran que en este artículo no aparecen datos de pacientes.

\section{Conflictos de interés}

Los autores declaran no tener ningún conflicto de interés.

\section{Bibliografía}

1. Rosanio S, Schwarz ER, Ware DL, Vitarelli A. Syncope in adults: systematic review and proposal of a diagnostic and therapeutic algorithm. Int J Cardiol. 2013;162(3):149-57. Epub 2011/12/23

2. Soteriades ES, Evans JC, Larson MG, Chen MH, Chen L, Benjamin EJ, et al. Incidence and prognosis of syncope. N Eng J Med. 2002;347(12):878-85. Epub 2002/09/20.

3. Edvardsson N, Frykman V, van Mechelen R, Mitro P, MohiiOskarsson A, Pasquie JL, et al. Use of an implantable loop recorder to increase the diagnostic yield in unexplained syncope: results from the PICTURE registry. Europace. 2011;13(2): 262-9. Epub 2010/11/26.

4. Brignole M, Menozzi C, Bartoletti A, Giada F, Lagi A, Ungar A, et al. A new management of syncope: prospective systematic guideline-based evaluation of patients referred urgently to general hospitals. Eur Heart J. 2006;27(1):76-82. Epub 2005/11/08

5. Jardine DL. Vasovagal syncope: new physiologic insights. Cardiol Clin. 2013;31(1):75-87. Epub 2012/12/12.

6. Linzer M, Yang EH, Estes NA, Wang P, Vorperian VR, Kapoor WN. Diagnosing syncope. Part 1: Value of history, physical examination, and electrocardiography. Clinical Efficacy Assessment Project of the American College of Physicians. Ann Int Med. 1997;126(12):989-96. Epub 1997/06/15.

7. van Dijk N, Boer KR, Colman N, Bakker A, Stam J, van Grieken JJ, et al. High diagnostic yield and accuracy of history, physical examination, and ECG in patients with transient loss of consciousness in FAST: the Fainting Assessment study. J Cardiovasc Electrophysiol. 2008;19(1):48-55. Epub 2007/ $10 / 06$.

8. Epstein AE, DiMarco JP, Ellenbogen KA, Estes NA, Freedman RA, Gettes LS, et al. ACC/AHA/HRS 2008 Guidelines for DeviceBased Therapy of Cardiac Rhythm Abnormalities: a report of the American College of Cardiology/American Heart Association Task Force on Practice Guidelines (Writing Committee to Revise the ACC/AHA/NASPE 2002 Guideline Update for Implantation of Cardiac Pacemakers and Antiarrhythmia Devices) developed in collaboration with the American Association for Thoracic
Surgery and Society of Thoracic Surgeons. J Am Coll Cardiiol. 2008;51(21):e1-62. Epub 2008/05/24.

9. Sud S, Massel D, Klein GJ, Leong-Sit P, Yee R, Skanes AC, et al. The expectation effect and cardiac pacing for refractory vasovagal syncope. Am J Med. 2007;120(1):54-62. Epub 2007/01/09.

10. Fitzpatrick A, Theodorakis G, Ahmed R, Williams T, Sutton R. Dual chamber pacing aborts vasovagal syncope induced by headup 60 degrees tilt. Pacing and clinical electrophysiology: PACE. 1991;14(1):13-9. Epub 1991/01/01.

11. Connolly SJ, Sheldon R, Roberts RS, Gent M. The North American Vasovagal Pacemaker Study (VPS). A randomized trial of permanent cardiac pacing for the prevention of vasovagal syncope. J Am Coll Cardiol. 1999;33(1):16-20. Epub 1999/ 02/06.

12. Sutton R, Brignole M, Menozzi C, Raviele A, Alboni P, Giani $P$, et al. Dual-chamber pacing in the treatment of neurally mediated tilt-positive cardioinhibitory syncope: pacemaker versus no therapy: a multicenter randomized study. The Vasovagal Syncope International Study (VASIS) Investigators. Circulation. 2000;102(3):294-9. Epub 2000/07/19.

13. Connolly SJ, Sheldon R, Thorpe KE, Roberts RS, Ellenbogen KA, Wilkoff BL, et al. Pacemaker therapy for prevention of syncope in patients with recurrent severe vasovagal syncope: Second Vasovagal Pacemaker Study (VPS II): a randomized trial. JAMA. 2003;289(17):2224-9. Epub 2003/ 05/08.

14. Raviele A, Giada F, Menozzi C, Speca G, Orazi S, Gasparini G, et al. A randomized, double-blind, placebo-controlled study of permanent cardiac pacing for the treatment of recurrent tilt-induced vasovagal syncope. The vasovagal syncope and pacing trial (SYNPACE). Eur Heart J. 2004;25(19):1741-8. Epub 2004/09/29.

15. Brignole M, Menozzi C, Moya A, Andresen D, Blanc JJ, Krahn $A D$, et al. Pacemaker therapy in patients with neurally mediated syncope and documented asystole: Third International Study on Syncope of Uncertain Etiology (ISSUE-3): a randomized trial. Circulation. 2012;125(21):2566-71. Epub 2012/ 05/09.

16. Lau CP, Tse HF, Camm AJ, Barold SS. Evolution of pacing for bradycardias: sensors. Eur Heart J. 2007;9 Suppl I: I11-22.

17. Occhetta E, Bortnik M, Audoglio R, Vassanelli C, INVASY Study investigators. Closed loop stimulation in prevention of vasovagal syncope. Inotropy controlled pacing in vasovagal syncope (INVASY): a multicentre randomized, single blind, controlled study. Europace. 2004;6:538-47.

18. Palmisano P, Zaccaria M, Luzzi G, Nacci F, Anaclerio M, Favale S. Closed-loop cardiac pacing vs. conventional dualchamber pacing with specialized sensing and pacing algorithms for syncope prevention in patients with refractory vasovagal syncope: results of a long-term follow-up. Europace: European pacing, arrhythmias, and cardiac electrophysiology. Journal of the working groups on cardiac pacing, arrhythmias, and cardiac cellular electrophysiology of the European Society of Cardiology. 2012;14(7):1038-43. Epub 2012/ $01 / 17$.

19. Bortnik M, Occhetta E, Dell'Era G, Secco GG, Degiovanni A, Plebani L, et al. Long-term follow-up of DDDR closed-loop cardiac pacing for the prevention of recurrent vasovagal syncope. J Cardiovasc Med. (Hagerstown, Md). 2012;13(4):242-5. Epub 2012/03/01.

20. Kanjwal K, Karabin B, Kanjwal Y, Grubb BP. Preliminary observations on the use of closed-loop cardiac pacing in patients with refractory neurocardiogenic syncope. Journal of interventional cardiac electrophysiology: an international journal of arrhythmias and pacing. 2010;27(1):69-73. Epub 2009/ $11 / 26$. 
21. Russo V, Rago A, Papa AA, Golino P, Calabro R, Russo MG, et al. The effect of dual-chamber closed-loop stimulation on syncope recurrence in healthy patients with tilt-induced vasovagal cardioinhibitory syncope: a prospective, randomised, single-blind, crossover study. Heart (British Cardiac Society). 2013;99(21):1609-13. Epub 2013/06/01.
22. van Dijk N, Boer KR, Wieling W, Linzer M, Sprangers MA. Reliability, validity and responsiveness of the syncope functional status questionnaire. J Gen Int Med. 2007;22(9):1280-5. Epub 2007/07/05. 